University of Wollongong

Research Online

Australian Institute for Innovative Materials -

Papers

Australian Institute for Innovative Materials

$1-1-2013$

\title{
Magnetic phase diagram and correlation between metamagnetism and superconductivity in Ru0.9Sr2YCu2.107.9
}

\author{
Rashmi Nigam \\ University of Wollongong, rnigam@uow.edu.au \\ Shane J. Kennedy \\ Australian Nuclear Science and Technology Organization \\ Alexey V. Pan \\ University of Wollongong, pan@uow.edu.au \\ SX. Dou \\ University of Wollongong, shi@uow.edu.au
}

Follow this and additional works at: https://ro.uow.edu.au/aiimpapers

Part of the Engineering Commons, and the Physical Sciences and Mathematics Commons 


\title{
Magnetic phase diagram and correlation between metamagnetism and superconductivity in Ru0.9Sr2YCu2.107.9
}

\author{
Abstract \\ The magnetic superconductor $\mathrm{Ru}_{0.9} \mathrm{Sr}_{2} \mathrm{YCu}_{2.1} \mathrm{O}_{7.9}$ (Ru-1212Y) has been investigated using neutron \\ diffraction under variable temperature and magnetic field. With the complementary information from \\ magnetization measurements, we propose a magnetic phase diagram $T-H$ for the Ru- 1212 system. \\ Uniaxial antiferromagnetic (AFM) order of $1.2 \mu_{B} /$ Ru atoms with moments parallel to the $c$-axis is found \\ below the magnetic transition temperature at $\sim 140 \mathrm{~K}$ in the absence of magnetic field. In addition, \\ ferromagnetism (FM) in the $a b$-plane develops below $~ 120 \mathrm{~K}$, but is suppressed at lower temperature by \\ superconducting correlations. Externally applied magnetic fields cause Ru-moments to realign from the \\ $c$-axis to the $a b$-plane, i.e. along the $\{1.1 .0\}$ direction, and induce ferromagnetism in the plane with $\sim 1 \mu$ Bat \\ $60 \mathrm{kOe}$. These observations of the weak ferromagnetism suppressed by superconductivity and the field- \\ induced metamagnetic transition between AFM and FM demonstrate not only competing orders of \\ superconductivity and magnetism, but also suggest a certain vortex dynamics contributing to these \\ magnetic transitions.
}

\section{Keywords}

metamagnetism, between, correlation, diagram, phase, magnetic, 1o7, 9, 9sr2ycu2, ru0, superconductivity

\section{Disciplines}

Engineering | Physical Sciences and Mathematics

\section{Publication Details}

Nigam, R., Kennedy, S. J., Pan, A. V. \& Dou, S. Xue. (2013). Magnetic phase diagram and correlation between metamagnetism and superconductivity in Ru0.9Sr2YCu2.107.9. European Physical Journal B: Condensed Matter and Complex Systems, 86: 280. 


\title{
Magnetic phase diagram and correlation between metamagnetism and superconductivity in $\mathrm{Ru}_{0.9} \mathrm{Sr}_{2} \mathrm{YCu}_{2.1} \mathrm{O}_{7.9}$
}

\author{
R. Nigam ${ }^{1}$, S. J. Kennedy ${ }^{2}$, A. V. Pan ${ }^{1, *}$, and S. X. Dou ${ }^{1}$ \\ ${ }^{1}$ Institute for Superconducting and Electronic Materials, University of Wollongong, \\ Northfields Avenue, Wollongong, NSW 2522, Australia and \\ ${ }^{2}$ Bragg Institute, Australian Nuclear Science and Technology Organization, \\ New Illawarra Road, Lucas Heights, NSW 2234, Australia
}

(Dated: 19 April 2013)

\begin{abstract}
The magnetic superconductor $\mathrm{Ru}_{0.9} \mathrm{Sr}_{2} \mathrm{YCu}_{2.1} \mathrm{O}_{7.9}$ (Ru-1212Y) has been investigated using neutron diffraction under variable temperature and magnetic field. With the complementary information from magnetization measurements, we propose a magnetic phase diagram $T-H$ for the Ru-1212 system. Uniaxial antiferromagnetic (AFM) order of $1.2 \mu_{B} / \mathrm{Ru}$ atoms with moments parallel to the $c$-axis is found below the magnetic transition temperature at $\sim 140 \mathrm{~K}$ in the absence of magnetic field. In addition, ferromagnetism $(\mathrm{FM})$ in the $a b$-plane develops below $\sim 120 \mathrm{~K}$, but is suppressed at lower temperature by superconducting correlations. Externally applied magnetic fields cause Ru-moments to realign from the $c$-axis to the $a b$-plane, i.e. along the $\langle 1,1,0\rangle$ direction, and induce ferromagnetism in the plane with $\sim 1 \mu_{B}$ at $60 \mathrm{kOe}$. These observations of the weak ferromagnetism suppressed by superconductivity and the field-induced metamagnetic transition between AFM and FM demonstrate not only competing orders of superconductivity and magnetism, but also suggest a certain vortex dynamics contributing to these magnetic transitions.
\end{abstract}

Published in Eur. Phys. J. B 86, 280 (2013).

*Email: pan@uow.edu.au (Corresponding author).

PACS numbers: 75.25.-j (Spin arrangements in magnetically ordered materials), 74.25.Ha (Magnetic properties including vortex structures and related phenomena)

\section{INTRODUCTION}

Coexistence of two mutually exclusive spin ordering phenomena, superconductivity (SC) and magnetism has sparked the interest in high temperature cuprate superconductors, $\mathrm{RuSr}_{2} \mathrm{RCu}_{2} \mathrm{O}_{8-\delta}(\mathrm{Ru}-1212)^{1-6}$ ( $\mathrm{R}$ indicates a rare earth element), where, the $\mathrm{RuO}_{2}$ layer is responsible for magnetic order and the $\mathrm{CuO}_{2}$ layer gives rise to superconductivity. Originally, bulk magnetization measurements ${ }^{1,7,8}$ and nuclear magnetic resonance $(\mathrm{NMR})^{9}$ reported weak-ferromagnetic order in the system. These results caught the attention of scientists because the presence of ferromagnetic (FM) exchange fields are expected to act on $\mathrm{CuO}_{2}$ layers and prevent the singlet-pair formation due to induced splitting of spin-up and spin-down conduction bands, thus inhibiting the occurrence of the superconducting state.

However, the presence of FM order in Ru-1212 has always been a debatable issue. Magnetization measurements can provide rather unambiguous indication of at least two ferromagnetic phase mixture ${ }^{4}$, which creates grounds for a diversity of magnetic transitions and states due to various form of spin and/or charge exchanges as considered in Refs. ${ }^{10-12}$. The neutron powder diffraction (NPD) studies reported in Ref. ${ }^{2}$ did not observe FM order in zero field, whereas NPD measurements in Ref. ${ }^{13}$ observed a FM component of $0.28 \mu_{B}$. Another NPD study ${ }^{14,15}$ attributed a small FM component arising from FM ordering of $\mathrm{CuO}_{2}$ planes that are antiferromagnetically stacked at temperatures below the mag- netic ordering temperature of $\mathrm{Ru}$ atoms $\left(T_{M} \sim 140 \mathrm{~K}\right)$. Recently, a representation analysis ${ }^{16}$ has proposed that a net in-plane FM component must be present, albeit compensated due to alternation of moment direction along the $c$-axis. This is in agreement with previous $\mathrm{NMR}^{9}$ and ferromagnetic resonance $(\mathrm{FMR})^{17}$ results. The strong indication of the G-type AFM ground state in Ru-1212 below Ru-magnetic ordering temperature has been obtained by NPD in Refs. ${ }^{2,13-15}$ and by x-ray resonance magnetic scattering (XRMS) ${ }^{16}$ measurements. However, XRMS indicated orientation of AFM moments along a lower-symmetry $\langle 1,0,2\rangle$ crystallographic direction with a substantial in-plane component ${ }^{16}$, whereas NPD studies ${ }^{2,13-15}$ have suggested alignment along the $c$-axis.

Indeterminacy regarding the magnetic behaviour of Ru-1212 has so far made it difficult to assess the influence of the surprisingly complex magnetic structure on the superconducting properties of the $\mathrm{CuO}_{2}$ layers. In this work, by combining the results obtained by neutron diffraction and magnetization $(M)$ measurements, we derive a $T-H$ phase diagram that effectively explains all features of magnetic and superconducting states in the magnetization curves of the $\mathrm{Ru}-1212$ system. We also focus on understanding the effect of intrinsic $\mathrm{Ru}$ magnetization on the SC state of the system. One way to probe this correlation is to trace the structural, magnetic and superconducting phase transformation on application of external magnetic field. Therefore, we have performed an accurate mapping of the magnetic structure in $\mathrm{Ru}_{0.9} \mathrm{Sr}_{2} \mathrm{YCu}_{2.1} \mathrm{O}_{7.9}(\mathrm{Ru}-1212 \mathrm{Y})$ sample ${ }^{5,18}$ as a 


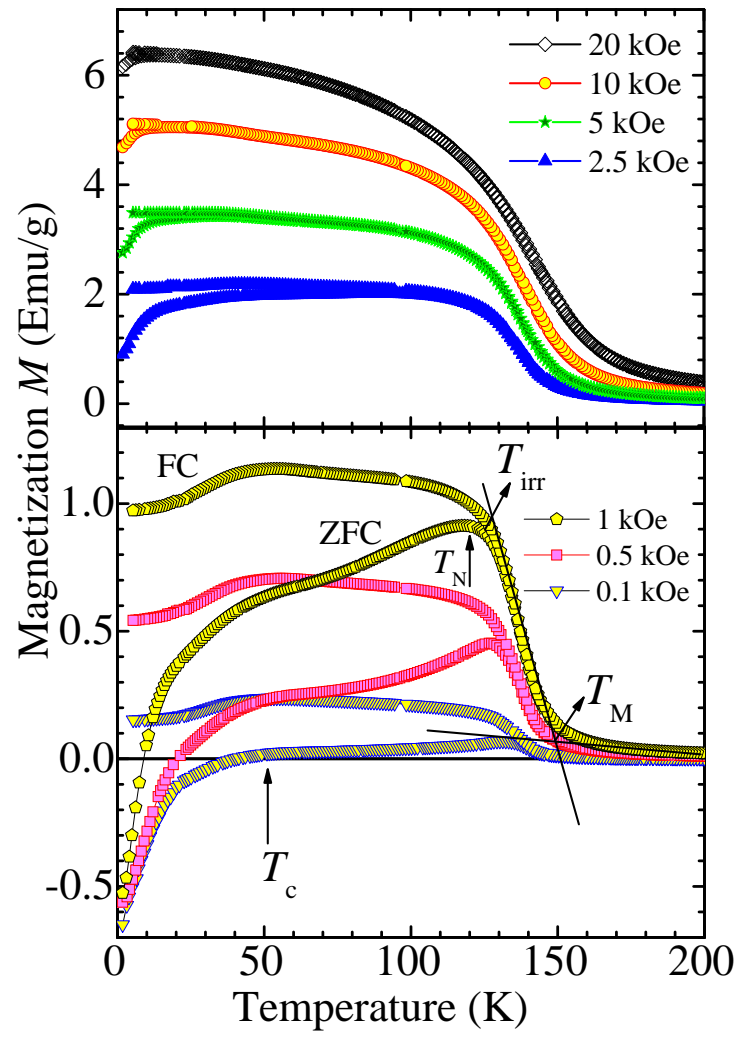

FIG. 1: Temperature dependence of zero-field cooled (ZFC) and field-cooled (FC) magnetization curves of $\mathrm{Ru}_{0.9} \mathrm{Sr}_{2} \mathrm{YCu}_{2.1} \mathrm{O}_{7.9}$ at different applied dc magnetic fields.

function of applied magnetic field in the SC state with the help of systematic NPD measurements. Experimental results demonstrate the affect of intrinsic Ru magnetization on vortex dynamics in the SC state, as well as provide evidence that the $\mathrm{SC}$ order depends on whether the $\mathrm{Ru}$ magnetization is parallel or perpendicular to the $\mathrm{RuO}_{2}$ layers, as predicted by Pickett et al. ${ }^{19}$ using density function theory.

\section{EXPERIMENTAL DETAILS}

A polycrystalline sample of $\mathrm{Ru}_{0.9} \mathrm{Sr}_{2} \mathrm{YCu}_{2.1} \mathrm{O}_{7.9}(\mathrm{Ru}-$ $1212 \mathrm{Y}$ ) was prepared by solid-state reaction using a high pressure $(\sim 6 \mathrm{GPa})$ and high temperature $(\sim 1450 \mathrm{~K})$ technique at the National Institute for Material Science (NIMS), Japan ${ }^{5,18}$. Our Rietveld refinement of the room temperature NPD pattern using FullProf ${ }^{20}$ software with tetragonal symmetry and space group $\mathrm{P} 4 / \mathrm{mmm}$, estimated the lattice parameters of the sample to be $a=$ $b=3.8098(3) \AA$, and $c=11.478(1) \AA$, which is consistent with other reports ${ }^{5,6}$.

DC magnetization measurements were performed using a Magnetic Properties Measurement System (Quantum Design) over the temperature range from 1.9 to 300 K. NPD measurements were carried out with the neu-

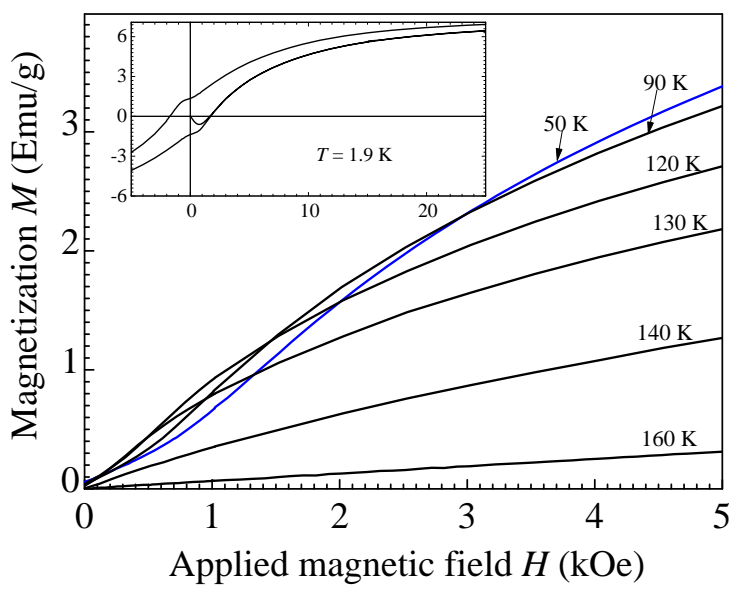

FIG. 2: Virgin magnetization curves as a function of applied magnetic field at different temperatures. The inset shows the magnetization versus applied field curve at $1.9 \mathrm{~K}$, exhibiting the diamagnetic Meissner-like signal for the virgin curve.

tron wavelength of $2.41 \AA$ on the high intensity powder diffractometer (Wombat) at the OPAL research reactor at the Australian Nuclear Science and Technology Organization (ANSTO). Temperature dependent NPD data was collected from $2 \theta=16^{\circ}$ to $136^{\circ}$ with a $1 \mathrm{~K}$ temperature interval between $10-160 \mathrm{~K}$ in the absence of magnetic field using a cryofurnace. NPD data was collected in the presence of magnetic field (0-64 kOe) at $10 \mathrm{~K}$ using a vertical field cryomagnet. The direction of applied field was perpendicular to the scattering vector. We had previously observed grain reorientation in loosely packed powders of $\mathrm{Ru}-1212 \mathrm{Y}$ in response to applied magnetic field $^{3}$, so in order to gain meaningful information, the orientation of crystallites was fixed by tightly clamping the powder sample.

\section{MAGNETIZATION MEASUREMENTS}

Fig. 1 shows the variation of the zero-field cooled $\left(M_{Z F C}\right)$ and field-cooled $\left(M_{F C}\right)$ magnetization of Ru$1212 \mathrm{Y}$ with temperature at different dc magnetic fields $(H)$. The $M_{Z F C}$ and $M_{F C}$ curves at 100 Oe rise sharply from non-magnetized state at $T_{M} \simeq 140 \mathrm{~K}$, indicating a transition from the paramagnetic (PM) to a magnetically ordered state. At the magnetic irreversibility temperature, $T_{i r r}<T_{M}$ the $M_{Z F C}$ and $M_{F C}$ curves bifurcates, suggesting the development of a frozen magnetic domain-state, which exhibits characteristics compatible with $\mathrm{AFM}$ and $\mathrm{FM}^{4}$. In the case of AFM ordering, the peak in the $M_{Z F C}$ curve would be characteristic of the Néel temperature $T_{N}$. Above $2.5 \mathrm{kOe}$, the irreversibility between the $M_{Z F C}$ and $M_{F C}$ curves is reduced, shifting 
$T_{i r r}$ to lower temperatures and the magnetization curves are more typical of FM behaviour.

The superconducting transition appears as a change in the slope of the $M_{Z F C}$ and $M_{F C}$ curves at $T_{c}$. This is particularly noticeable in the $M_{Z F C}(T)$ curves where a clear diamagnetic signal can be observed. At $H<1 \mathrm{kOe}$, $T_{c}$ is independent of the applied field being $\simeq 40 \mathrm{~K}$, whereas it shifts to lower temperatures with increasing $H$. Superconductivity is further verified by isothermal magnetization $[M(H)]$ curve measured at $1.9 \mathrm{~K}$, shown in the inset of Fig. 2 that exhibits a strong diamagnetic signal at low magnetic fields characteristic of the Meissner state. This behaviour is not present at $T>T_{c} \simeq 40 \mathrm{~K}$ (Fig. 2).

In general, similar results can be obtained in Ru-1212 even if $\mathrm{Y}$ is replaced by other rare earth elements, such as Gd. ${ }^{21}$ However, as mentioned in Sect. I, there were many different interpretations ${ }^{2,14-16}$ reviewed in Ref. ${ }^{4}$. In present work, we employ these results in combination with structural magnetic characterisations to derive a consistent magnetic phase diagram with corresponding structural transformations, resolving existing contradictions.

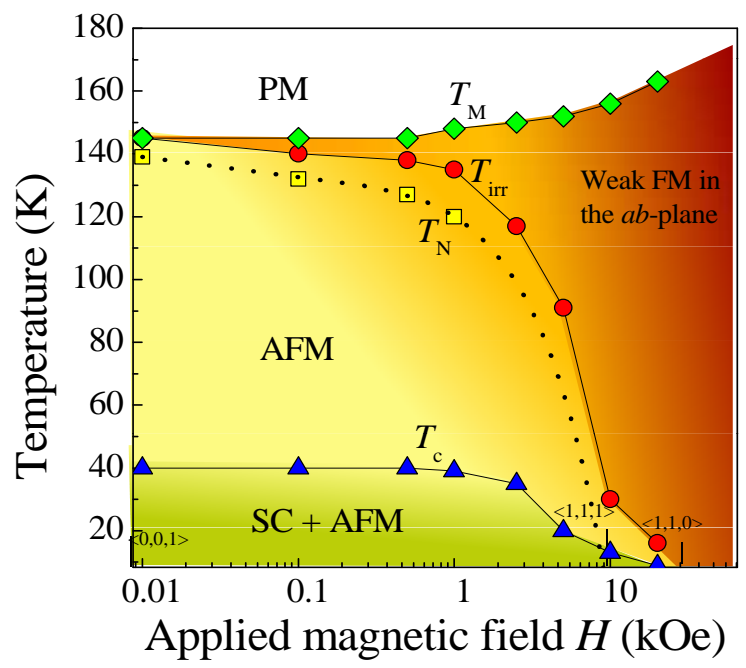

FIG. 3: Log-linear plot of the magnetic phase diagram of $\mathrm{Ru}_{0.9} \mathrm{Sr}_{2} \mathrm{YCu}_{2.1} \mathrm{O}_{7.9}$. The different magnetic states defined are paramagnetic (PM), antiferromagnetic (AFM), and weakferromagnetic (weak-FM). The superconducting state is denoted by SC. The colour gradient represents the gradual spin reorientation as the system progresses from the AFM state to the weak-FM state. Small vertical lines are the markers to show direction of spin orientation as a function of applied field at $10 \mathrm{~K}$. Note that the $T$-axis starts from $9 \mathrm{~K}$, which also means that $T_{c}$ does not disappear at the highest field of the measurements of $20 \mathrm{kOe}$, i.e. the $\mathrm{SC}$ ordering is expected to survive at higher magnetic fields.

\section{MAGNETIC PHASE DIAGRAM}

The $T-H$ magnetic phase diagram of Ru-1212Y derived from this work is summarized in Fig. 3. The phase boundaries shown in the magnetic phase diagram are determined from the characteristic temperatures $T_{\mathrm{M}}$ (diamond symbols), $T_{i r r}$ (circles), $T_{N}$ (squares), and $T_{c}$ (triangles), extracted from the $M_{Z F C}(T)$ and $M_{F C}(T)$ curves shown in Fig. 1.

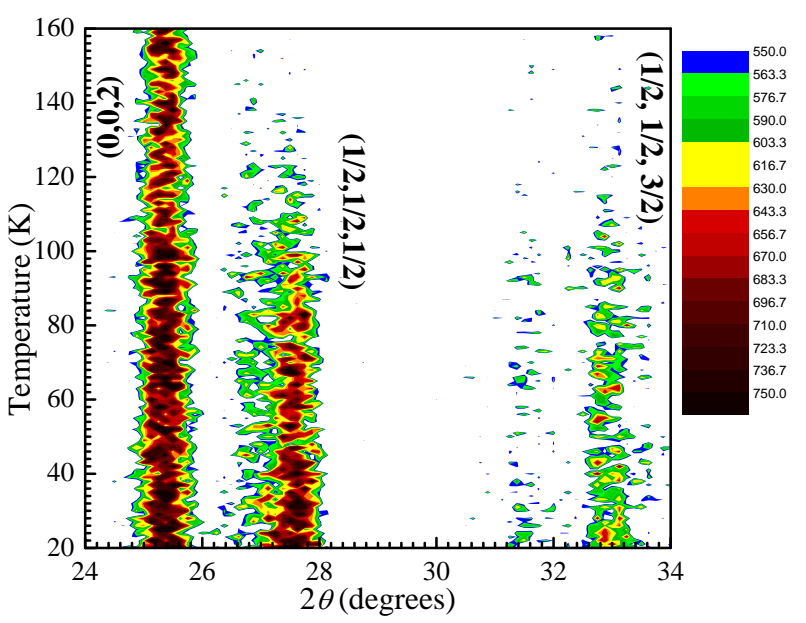

FIG. 4: $2 \mathrm{D}$ contour plot of low- $2 \theta$ portion of the neutron diffraction pattern measured as a function of temperature without application of magnetic field. The plot shows the $(0,0,2)$ nuclear Bragg peak at $2 \theta=25.4^{\circ}$, and the magnetic $\left(\frac{1}{2} \frac{1}{2} \frac{1}{2}\right)$ and $\left(\frac{1}{2}, \frac{1}{2}, \frac{3}{2}\right)$ peaks at $2 \theta=27.5^{\circ}$ and $33^{\circ}$, respectively.

The $T-H$ phase diagram in Fig. 3 shows that $T_{c}$ remains unchanged up to $H \simeq 1 \mathrm{kOe}$. Similarly, there is a little change observed in the magnetic ordering temperatures related to the $\mathrm{Ru}$ sublattice, i.e. $T_{\mathrm{M}}, T_{i r r}$, and $T_{N}$ below $H \simeq 1 \mathrm{kOe}$. Notably, $T_{c}$ starts to degrade at $H \geq 1 \mathrm{kOe}$, which is the same field at which $T_{i r r}$ and $T_{N}$ starts to decrease sharply. We note that García et al. $^{22}$ also observed change in the behaviour of the superconducting region at $H=1 \mathrm{kOe}$ in their field dependent resistivity and ac susceptibility measurements. In the phase diagram, the decreasing $T_{i r r}(H)$ and $T_{N}(H)$ curves in increasing field may indicate a metamagnetic transition separating AFM from FM state, likely stipulated by spin exchange interactions described in our phase mixture model ${ }^{4}$ and built upon in the following works ${ }^{10-12}$. The types of temperature and field dependent magnetic orderings shown in the phase diagram have been determined from the NPD measurements, which will be discussed in detail in Sections A and B. Correlation between magnetism and superconductivity will be explained in Section C. 


\section{A. Magnetic and superconducting response in the absence of applied magnetic field}

Fig. 4 displays a 2D-contour plot of the portion of the NPD pattern from $2 \theta=24^{\circ}$ to $34^{\circ}$ as a function of temperature at $H=0$ Oe. The figure shows that the nuclear Bragg peak $(0,0,2)$ at $2 \theta=25.4^{\circ}$ is accompanied by the two magnetic peaks $\left(\frac{1}{2} \frac{1}{2} \frac{1}{2}\right)$ and $\left(\frac{1}{2}, \frac{1}{2}, \frac{3}{2}\right)$ at $2 \theta=27.5^{\circ}$ and $33^{\circ}$ respectively below $140 \mathrm{~K}$. These magnetic peaks are indexed using propagation vector $\kappa=\left(\frac{1}{2}, \frac{1}{2}, \frac{1}{2}\right)$, which corresponds to the $G$-type AFM spin arrangement, where nearest neighbouring magnetic ions are antiferromagnetically coupled along the three crystallographic axes. The orientation of AFM moments was determined to be along $\langle 0,0,1\rangle$ direction as shown in Fig. 5. The spin alignment was concluded from the calculated ratio of the intensities of the $\left(\frac{1}{2}, \frac{1}{2}, \frac{1}{2}\right)$ and $\left(\frac{1}{2}, \frac{1}{2}, \frac{3}{2}\right)$ peaks, which was 2.25 , in close agreement with the observed intensity ratio $(\sim 2.1)$ of these two peaks measured at $10 \mathrm{~K}$ and $H=0$ Oe. We could find no other moment direction that produces a similar intensity ratio.

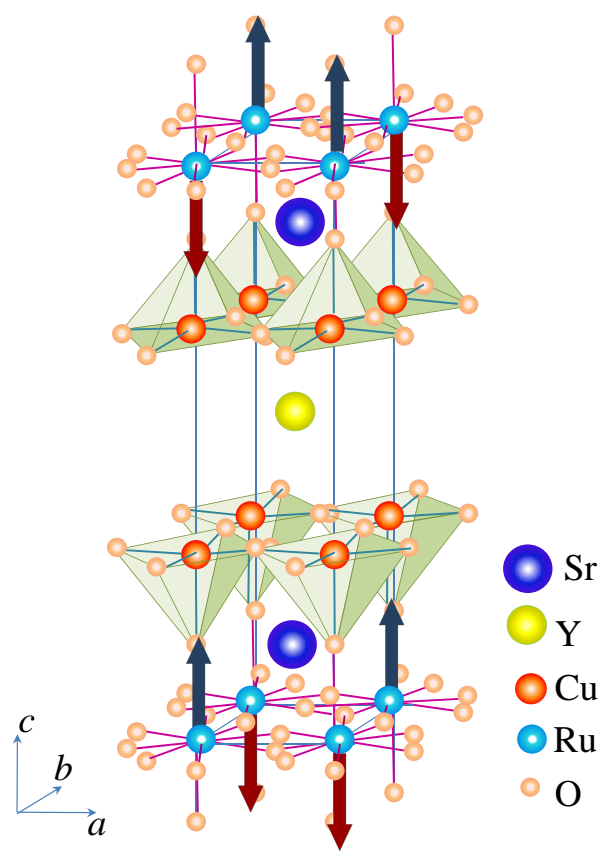

FIG. 5: Crystal structure of the unit cell of $\mathrm{Ru}_{0.9} \mathrm{Sr}_{2} \mathrm{YCu}_{2.1} \mathrm{O}_{7.9}$ determined from neutron powder diffraction measurements at $H=0$ Oe. The arrows show the direction of the Ru moments.

In addition to the observation of a stable AFM order in the SC state, an interesting observation has been made in the temperature dependence of the $\left(0,0, l_{\text {even }}\right)$ nuclear Bragg peaks. Fig. 6 shows that while the intensity of the $\left(\frac{1}{2}, \frac{1}{2}, \frac{1}{2}\right)$ peak increases with decreasing tem- perature, that of the nuclear $(0,0,2)$ peak increases below $\sim 110 \mathrm{~K}$, indicating emerging FM order. At around $90 \mathrm{~K}$, the NPD peaks contributing to the FM signal component of the $(0,0,2)$ peak intensity start to gradually decrease and disappear completely by $T_{c} \simeq 40 \mathrm{~K}$. We speculate that this observation is due to suppression of spontaneous FM by superconductive (SC) correlations starting from $T \sim 90 \mathrm{~K}$ (which may or may not be associated with the pseudogap formation above $T_{c}$ ). Given the close structural similarity between Ru-1212 and yttrium barium copper oxide (YBCO) superconductor with its $T_{c} \simeq 90 \mathrm{~K}$, this result may not be so surprising. Based on these data we estimate that an FM moment of $\approx 0.8$ $\mu_{B} / \mathrm{Ru}$ atom develops at $90 \mathrm{~K}$. Extrapolation of the mean field model $^{2}$ (solid curve in Fig. 6) suggests that by $20 \mathrm{~K}$ a FM moment of $\sim 1.2 \mu_{B} / \mathrm{Ru}$ atom would have developed in the absence of the SC order.

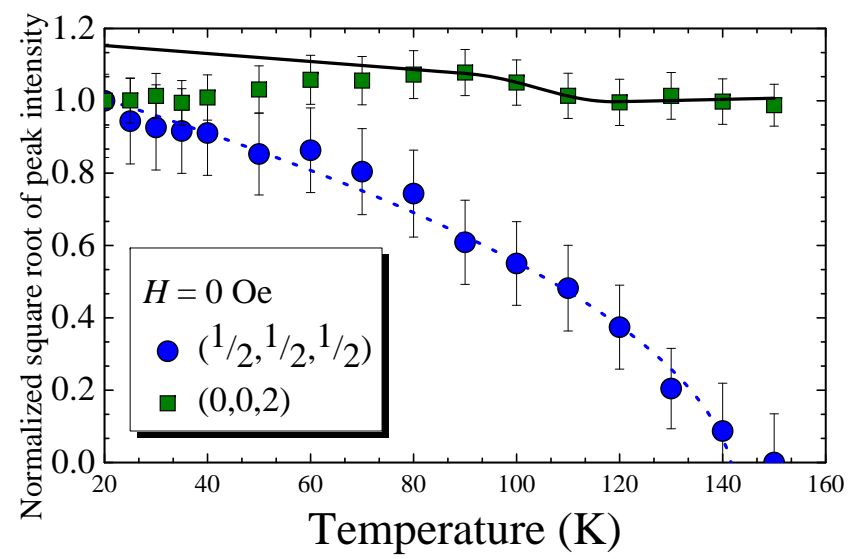

FIG. 6: Normalized square root of intensities of $(0,0,2) \mathrm{nu}-$ clear Bragg peak, and $\left(\frac{1}{2} \frac{1}{2} \frac{1}{2}\right)$ antiferromagnetic peak (extracted from Gaussian peak fitting) as a function of temperature. The dotted curve shows the fit to the mean-field theory, estimating a Néel temperature of $142.6 \mathrm{~K}^{2}{ }^{2}$

\section{B. In-field magnetic response}

Fig. 7 shows a 2 D-contour plot of the low- $2 \theta$ portion of the NPD pattern measured at $10 \mathrm{~K}$, as a function of applied magnetic field. With increasing $H$, the intensity of the AFM peaks is reduced, but does not completely disappear even up to $64 \mathrm{kOe}$. Fig. 8 shows the normalized intensities of $(0,0,2),(0,0,3)$, and $(1,0,3)$ nuclear peaks and the $\left(\frac{1}{2}, \frac{1}{2}, \frac{1}{2}\right)$ magnetic peak as a function of $H$. A rather sharp decrease in the intensity of $\left(\frac{1}{2}, \frac{1}{2}, \frac{1}{2}\right)$ can be observed up to $\sim 20 \mathrm{kOe}$, but thereafter the intensity gradually levels off. In contrast, the intensity of the magnetic response increases for the $(0,0,2)$ reflection, producing a gradual rise up to $13 \mathrm{kOe}$ and reducing in slope thereafter. A similar trend was observed in other 
$\left(0,0, l_{\text {even }}\right)$ peak intensities as well. We also note that there is small significant growth in the intensity of the $(0,0,3)$ peak with field, but not in the $(1,0,3)$ peak intensity.

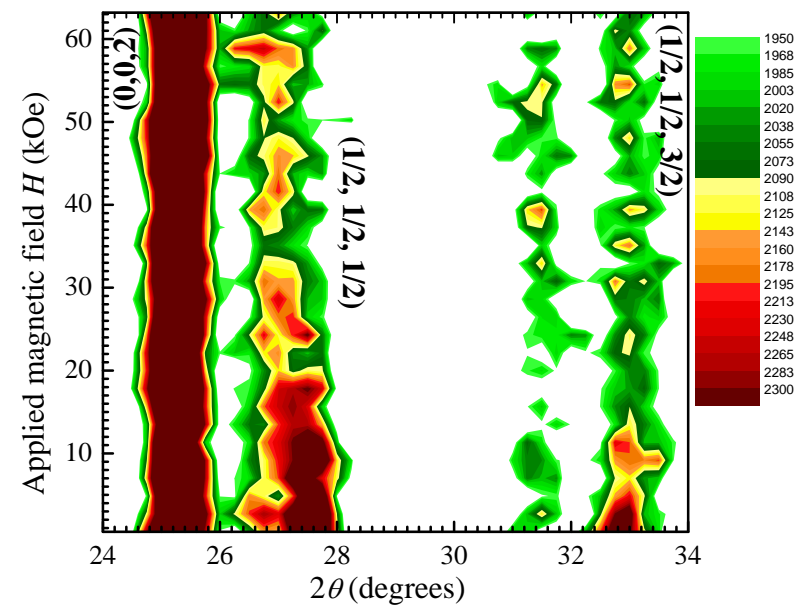

FIG. 7: 2D contour plot of the low- $2 \theta$ portion of the neutron diffraction pattern measured at $10 \mathrm{~K}$ as a function of the applied magnetic field. The plot shows the $(0,0,2)$ nuclear Bragg peak, and the magnetic $\left(\frac{1}{2} \frac{1}{2} \frac{1}{2}\right)$ and $\left(\frac{1}{2}, \frac{1}{2}, \frac{3}{2}\right)$ peaks.

These experimental observations were mathematically simulated by rotating $\mathrm{Ru}$ AFM moment from the initial $c$-axis orientation to the plane perpendicular to it, while testing several different axes through which Ru spin could possibly rotate. Fig. 8 shows observed (symbols) and cal-

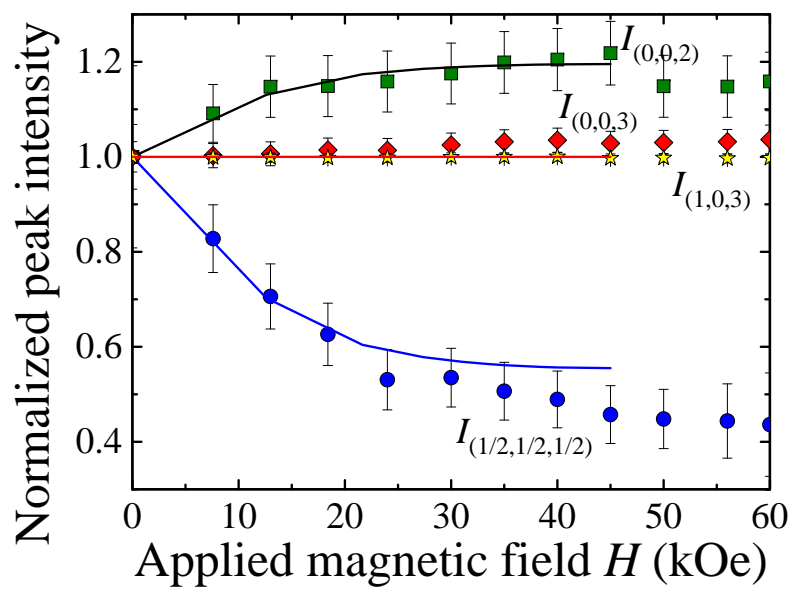

FIG. 8: Normalized intensities of the $(0,0,2),(0,0,3)$ and $(1,0,3)$ nuclear Bragg peaks, as well as the $\left(\frac{1}{2}, \frac{1}{2}, \frac{1}{2}\right)$ magnetic peak of Ru-1212Y at $10 \mathrm{~K}$ as a function of the applied magnetic field. The solid lines are the calculated intensities of the $(0,0,2)$ and $\left(\frac{1}{2}, \frac{1}{2}, \frac{1}{2}\right)$ peaks, fitting the respective observed intensities as the Ru spin rotates from $\langle 0,0,1\rangle$ at zero-field to $\langle 1,1,0\rangle$ at $45 \mathrm{kOe}$, while passing through the direction of propagation $(1,1,1)$ at $13 \mathrm{kOe}$. culated (solid lines) intensities for $\left(\frac{1}{2}, \frac{1}{2}, \frac{1}{2}\right)$ and $(0,0,2)$ as a function of $H$. A good agreement between observed and calculated intensities was reached by rotating the Ru AFM spins with moment of $1.2 \mu_{B}$ from the $\langle 0,0,1\rangle$ orientation to $\langle 1,1,0\rangle$, while indexing the rotation through the $\langle 1,1,1\rangle$ propagation vector in such a way that the tetragonal symmetry was maintained (Fig. 9). The growth of the $\left(0,0, l_{\text {odd }}\right)$ intensities suggests the growth of FM component in the $a b$-plane (i.e. parallel to the applied field and perpendicular to the scattering vector). This component is independent of rotations of AFM constituent. The apparent absence of growth of the $(1,0,3)$ intensity is only due to the relative strength of the nuclear component of $(1,0,3)$.

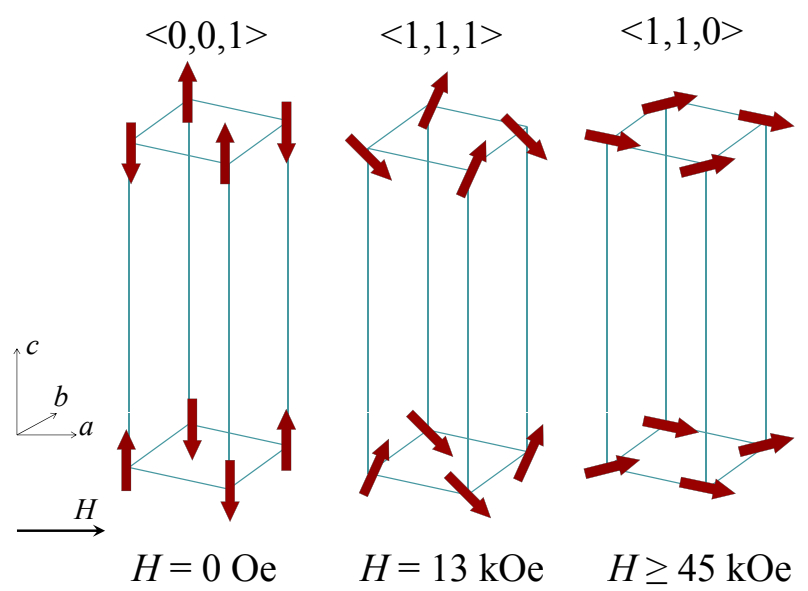

FIG. 9: Magnetic structure of $\mathrm{Ru}-1212 \mathrm{Y}$ with spin orientation as a function of the applied magnetic field at $T=10 \mathrm{~K}$. The solid $H$-arrow shows the direction of the field.

The mechanism governing the response of $\mathrm{Ru}$ moments to the applied field could be understood as follows. The magnetic interactions below $T_{N}$ at $H=0$ Oe remain strongly coupled till $\sim 1 \mathrm{kOe}$ resulting in only a small degree of spin reorientation and hence a slight change in $T_{i r r}$ and $T_{N}$ as shown in the phase diagram in Fig. 3. However, at high $H$, the Zeeman energy becomes strong enough to disturb and slightly reduce the AFM coupling (likely leading to about 20\% difference between the experiment and the fit of the $\left(\frac{1}{2} \frac{1}{2} \frac{1}{2}\right)$ peak intensity in Fig. 8). AFM moments rotate towards the direction of $H$. Overall, this results in gradual vanishing the AFM peak in $M_{Z F C}(T)$ and in more reversible behaviour between $M_{Z F C}$ and $M_{F C}$ (Fig. 1) at higher fields, which is likely to drive the entire system toward a (weak) FM state with a net moment of $1.2 \mu_{B} / \mathrm{Ru}$ atom in the $a b$ plane. According to NPD results (Fig. 7), this process of magnetic moment rotations is rather gradual in increasing field. However, it is in a sharp contrast to the spin-flop rotation into another AFM structure observed in Refs. ${ }^{2,22}$. In Fig. 3, this gradual process is represented 
by the colour gradient, which is cut through by the metamagnetic transition at $T_{N}$ from AFM to weak-FM. One of the most probable reasons for the observed metamagnetism in Ru-1212 could be the weak connection between the magnetic $\mathrm{RuO}_{2}$ layers due to the crystal anisotropy with lattice parameter $c>>a$. This would reduce the AFM exchange interactions between two layers, causing a relatively low field strength of $1 \mathrm{kOe}$ to result in the metamagnetic transition.

At large fields, the entire system would form large FM domains, which would have larger relaxation times resulting in de-freezing of the domain state at higher temperatures. This behaviour is observed in the $T_{\mathrm{M}}$ dependence at $H>1 \mathrm{kOe}$ in Fig. 3.

\section{Correlation between superconductivity and magnetism}

As described above, our magnetization measurements unambiguously indicate the superconducting properties below $T_{c} \simeq 40 \mathrm{~K}$, whereas the AFM magnetic ordering co-existing in the SC state is clearly shown by NPD results. This co-existence of the competing orderings is possible in neighbouring atomic layers of the natural multilayered system.

A striking correlation has been found between the SC order and the direction of the Ru magnetization, which corroborates the theoretical studies of Pickett et al. ${ }^{19}$. The phase diagram shows that the SC transition temperature $\left(T_{c}\right)$ is constant below $H \simeq 1 \mathrm{kOe}$. Within this low field range, the AFM ordering of $\mathrm{Ru}$ moment along $c$-axis gives rise to internal (dipole) field $B_{\text {int }}=4 \pi M$ (ref. ${ }^{19}$ ) within $\mathrm{RuO}_{2}$ planes, which results in $\mathrm{Ru}$ magnetization being perpendicular to $\mathrm{CuO}_{2}$ planes. As $H \neq 0$, the total effective field to consider is $H+B_{\text {int }}$. However, the (spontaneous) vortex state would be generated only by $B_{\text {int }}$ component as long as $H \leq B_{\text {int }}$ and negligible Zeeman splitting, preventing additional vortices due to $H$ from entering the sample. Indeed, the $\mathrm{Ru}$ moment of $1.2 \mu_{B}$ deduced from NPD measurements would generate $B_{\text {int }} \simeq 800$ Oe just as predicted in Ref. ${ }^{19}$. This also coincides remarkably well with the value of the external field at which $T_{c}$ start to degrade (Fig. 3), implying that additional vortices start to penetrate the sample at $H>B_{\text {int }} \simeq 800 \mathrm{Oe}$ (provided that $H<H_{c 1}$ as seen in the inset to Fig. 2). As a result, $T_{c}$ starts to degrade likely due to excessive perturbations into the orbital coupling within superconducting layers. Hence, the lower temperatures are needed in increasing fields above $B_{\text {int }}$ to ensure supercurrents for Meissner-like diamagnetic signal observed $M_{Z F C}(T)$ curves, which is shifted to lower temperatures (Fig. 1).

For $H>>B_{\text {int }}$, the effect of the intrinsic magnetization becomes progressively less important as the flux induced by external magnetic fields becomes dominant. High external fields not only turn the Ru magnetization in-plane, as shown by the neutron data, but also moves the system towards the upper critical field $H_{c 2}$ resulting in disappearance of Meissner-like state (Fig. 1) and eventually in collapsing superconductivity.

These experimental results are in agreement with the calculations done by Pickett et al. ${ }^{19}$ using density functional theory that showed that the dipolar and exchange fields are weak enough in $\mathrm{Ru}-1212$. Therefore, singlet pairing can still occur in the $\mathrm{CuO}_{2}$ layers with a modulated SC order parameter, while the pairing depends on whether the $\mathrm{Ru}$ magnetization is parallel or perpendicular to the $\mathrm{CuO}_{2}$ layers.

\section{CONCLUSION}

The impact of magnetic behaviour on the superconducting properties of $\mathrm{Ru}_{0.9} \mathrm{Sr}_{2} \mathrm{YCu}_{2.1} \mathrm{O}_{7.9}$ has been explained by our $T-H$ phase diagram, which has been derived from dc magnetization and neutron powder diffraction measurements. In the absence of the applied magnetic field, the system is likely in the $G$-type antiferromagnetic state with $\mathrm{Ru}$ magnetic moments oriented along the $c$-axis below $T_{N} \simeq 140 \mathrm{~K}$. Notably, NPD results do not exhibit any FM ordering in the SC state at the highest magnetic fields $(\sim 10 \mathrm{kOe})$ at which the $\mathrm{SC}$ state still survived likely due to screening out the effect of the external field. In contrast, above $T_{c}$ upon applying external magnetic field, the $\mathrm{Ru}$ moments rotate from their initial $\langle 0,0,1\rangle$ orientations to $\langle 1,1,0\rangle$ as indexed via the propagation vector $\langle 1,1,1\rangle$. Within the temperature range of $40 \mathrm{~K}$ to $\sim 110 \mathrm{~K}$, the characteristic of FM ordering has been found, which develops into the metamagnetic transition from the predominantly $c$-axis oriented AFM state towards the in-plane weak ferromagnetic state at fields of around $1 \mathrm{kOe}$ (being slightly temperature dependent). A remarkable agreement has been found between the experimentally observed onset of the degradation of spontaneous internal vortex state at $H \simeq 800$ Oe (reflected by degrading $T_{c}(H)$ from the constant value at smaller fields) due to the $\mathrm{Ru}$ magnetization and the values predicted theoretically ${ }^{19}$. Notably, the superconducting properties and vortex dynamics of the system have been found to be strongly affected by the Ru magnetization being either parallel or perpendicular to the superconducting $\mathrm{CuO}_{2}$ planes, whose orientation in turn depends on the strength of the field applied.

\section{ACKNOWLEDGMENT}

This work is supported by the Australian Research Council via A. V. Pan's Discovery Projects (DP0879933 and DP110100398) and AINSE Research Project No. P368. The authors would like to thank V. P. S. Awana from the National Physical Laboratory (New Delhi, India), E. Takayama-Muromachi and S. Balamurugan from the National Institute for Materials Science (NIMS, Japan) for providing the $\mathrm{Ru}_{0.9} \mathrm{Sr}_{2} \mathrm{YCu}_{2.1} \mathrm{O}_{7.9}$ sample. 
The authors have also benefited from fruitful discussions

with J. Wang and C. Ulrich.

${ }^{1}$ C. Bernhard, J. L. Tallon, Ch. Niedermayer, Th. Blasius, A. Golnik, E. Brucher, R. K. Kremer, D. R. Noakes, C. E. Stronach, and E. J. Ansaldo, Phys. Rev. B 59, 14099, (1999).

2 J. W. Lynn, B. Keimer, C. Ulrich, C. Bernhard, and J. L. Tallon, Phys. Rev. B 61, 14964 (2000).

${ }^{3}$ R. Nigam, A. V. Pan, S. J. Kennedy, A. J. Studer, N. Stuesser, and S. X. Dou, J. Appl. Phys. 107, 09E134 (2010).

${ }^{4}$ R. Nigam, A. V. Pan, S. X. Dou, Phys. Rev. B 77, 134509 (2008).

5 V. P. S. Awana and E. Takayama-Muromachii, Phys. Rev. B 67, 172502 (2003).

${ }^{6}$ O. Chmaissem, J. D. Jorgensen, H. Shaked, P. Dollar, and J. L. Tallon, Phys. Rev. B 61, 6401 (2000).

7 G. V. M. Williams and S. Krämer, Phys. Rev. B 62, 4132 (2000).

8 J. L. Tallon, J. W. Loram, G. V. M. Williams, and C. Bernhard, Phys. Rev. B 61, 6471 (2000).

9 Y. Tokunaga, H. Kotegawa, K. Ishida, Y. Kitaoka, H. Takagiwa, and J. Akimitsu, Phys. Rev. Lett. 86, 5767 (2001).

10 R. Nigam, A. V. Pan, and S. Dou, Eur. Phys. J. B 74, 429 (2010).

11 R. Nigam, A. V. Pan, and S. Dou, J. Phys.: Cond. Mater 23, 435702 (2011)

12 A. V. Pan, R. Nigam, and S. Dou, Physica C 470, 5707 (2010).

13 H. Takagiwa, J. Akimitsu, H. Kawano-Furukawa, and H. Yoshizawa, J. Phys. Soc. Jpn. 70, 333 (2001).

14 W. B. Yelon, Q. Cai, Jagat Lamsal, H. A. Blackstead, M. Kornecki, V. P. S. Awana, H. Kishan, S. Balamurugan, and E. Takayama-Muromachi, J. Appl. Phys. 101, 09G104 (2007).

15 H. A. Blackstead, W. B. Yelon, M. Kornecki, M. P. Smylie, Q. Cai, J. Lamsal, V. P. S. Awana, S. Balamurugan, and E. Takayama-Muromachi, Phys. Rev. B 76, 094507 (2007).

16 B. Bohnenbuck, I. Zegkinoglou, J. Strempfer, C. S. Nelson, H.-H. Wu, C. Schüssler-Langeheine, M. Reehuis, E. Schierle, Ph. Leininger, T. Herrmannsdörfer, J. C. Lang, G. Srajer, C. T. Lin, and B. Keimer, Phys. Rev. Lett. 102, 037205 (2009).

17 A. Butera, A. Fainstein, E. Winkler, J. Tallon, Phys. Rev. B 63, 054442 (2001).

18 V. P. S. Awana, T. Kawashima, and E. TakayamaMuromachii, Physica C 390, 101 (2003).

19 W. E. Pickett, R. Weht and A. B. Shick, Phys. Rev. Lett. 83, 3713 (1999).

20 Juan Rodríguez-Carvajal, Physica B 192, 55 (1993).

21 T. Nachtrab, C. Bernhard, C. Lin, D. Koelle, and R. Kleiner, C. R. Physique 7, 68 (2006).

22 S. García and L. Ghivelder, Phys. Rev. B 70, 052503 (2004).

23 A. C. Mclaughlin, I. Felner, and V. P. S. Awana, Phys. Rev. B 78, 094501 (2008).

${ }^{24}$ I. Felner, I. Nowik, M. I. Tsindlickt, O. Yuli, I. Asulin, O. Millo, V. P. S. Awana, H. Kishan, S. Balamurugan and E. Takayama-Muromachi, Phys. Rev. B 76, 144514 (2007).

25 C. S. Knee, B. D. Rainford, and M. T. Weller, J. Mater. Chem. 10, 2445 (2000).

26 J. W. Lynn, Y. Chen, Q. Huang, S. K. Goh, and G. V. M. Williams, Phys. Rev. B 76, 014519 (2007). 